\title{
Epilepsy in adult patients with Down syndrome: a clinical-video EEG study
}

\author{
Aglaia Vignoli ${ }^{1}$, Elena Zambrelli ${ }^{1}$, Valentina Chiesa ${ }^{1}$, \\ Miriam Savini ${ }^{1}$, Francesca La Briola ${ }^{1}$, Elena Gardella ${ }^{1}$, \\ Maria Paola Canevini ${ }^{1,2}$ \\ ${ }^{1}$ Centro Epilessia, Azienda Ospedaliera San Paolo, Milano \\ 2 Dipartimento di Medicina Chirurgia e Odontoiatria, Università degli Studi di Milano, \\ Italy
}

Received May 13, 2010; Accepted February 28, 2011

\begin{abstract}
Patients with Down syndrome are now living longer and the overall prevalence of epilepsy is increasing, however, full characterisation of epilepsy in adult age is still incomplete. We describe the electroclinical characteristics of epilepsy in 22 adult patients with Down syndrome (11 males, 11 females), with a mean age of 46 years (range: 28-64 years), followed at the Epilepsy Centre, San Paolo Hospital in Milan. Mean age at epilepsy onset was 36.8 years (range: 6-60 years). Nine out of 22 patients had focal epilepsy, while nine had late-onset myoclonic epilepsy. In four patients, epilepsy was unclassified. The EEG pattern of our patients was characterised by a progressive slowing of the background activity with sharp-and-slow waves with frontal predominance. In the patients diagnosed with late-onset myoclonic epilepsy, the EEGs showed generalised polyspike waves. Three subjects had an episode of myoclonic status epilepticus at the beginning or in the course of the disorder. After the first descriptions of lateonset myoclonic epilepsy by Genton and Paglia (1994), this is one of the largest patient cohorts reported. Our data confirm that epilepsy in adult patients with Down syndrome presents peculiar electroclinical characteristics which should be recognized early as prompt, effective treatment may be beneficial. [Published with video sequences]
\end{abstract}

Key words: Down syndrome, adult, epilepsy, late-onset myoclonic epilepsy (LOMEDS), focal seizures

Down syndrome (DS) (trisomy 21) is the most frequent chromosomal cause of mental retardation, with a frequency rate of about 1 in 700 births (Sherman et al., 2007). The prevalence of epilepsy in subjects with DS is reported to range from $1 \%$ to $13 \%$, higher than in the general population but lower than in patients with mental retardation
(Smigielska-Kuzia et al., 2009; Arya et al., 2011).

In DS, a bimodal distribution of seizure onset has been described: the first peak of incidence during early childhood and the second in middle age (Moller et al., 2001). The prevalence of epilepsy in patients with DS has also been reported to increase with age, with a prevalence 
of $46 \%$ in subjects over 50 years of age (Mc Vicker et al., 1994). Since life expectancy for subjects with DS has increased in the last decades, a larger overall proportion of them now have epilepsy.

Peculiar electroclinical patterns and epileptic syndromes have been recorded in adult patients with DS: focal epilepsies, reflex seizures (auditory stimulus) and myoclonic epilepsy associated with dementia (Genton and Paglia, 1994), defined as "Late-onset myoclonic epilepsy in Down syndrome" (LOMEDS) (Moller et al., 2001). The electroclinical features of LOMEDS are massive myoclonic jerks on awakening and generalised tonic-clonic seizures, with generalised polyspikes/spikes and waves on EEG and progressive dementia, usually preceding epilepsy onset (Crespel et al., 2007; Menéndez, 2005). Recently, Ferlazzo et al. described a group of DS patients with a peculiar type of Lennox-Gastaut syndrome, characterised by late onset and high incidence of reflex seizures (Ferlazzo et al., 2009).

In this study, we examined the electroclinical findings, epilepsy characteristics, relationship between seizure onset and cognitive decline and response of seizures to drug therapy in a cohort of patients with DS, evaluated over a 14 -year period at a single regional centre. The accompanying video documents seizure semiology in two patients.

\section{Patients and methods}

We retrospectively analysed all patients diagnosed with DS and epilepsy, aged more than 18 years, followed at our Centre between January 1995 and December 2009. The clinical diagnosis of DS based on typical somatic features was confirmed by cytogenetic analysis. Seizures were categorised according to the proposed classification of epileptic seizures and epilepsy by the Commission on Classification and Terminology of the ILAE, 2001 (Engel, 2001).

Patients were seen at least twice a year, with video-EEG polygraphic recording and neurological examination. Video-EEG polygraphic recordings were performed during wakefulness using a digital EEG System (Micromed, Treviso). Scalp electrodes were positioned according to the international 10/20 system with EMG electrodes for deltoid muscles. Neuroradiological and neuropsychological findings were recorded when available. The presence and characteristics of cognitive deterioration and behavioural problems were screened through parental/caregiver interview, focusing on the description of different behavioural patterns, including frequency and relationship with seizure onset. At the onset of cognitive deterioration, the patients were screened for other medical conditions which could cause such decline (e.g. thyroid dysfunction, metabolic disorders). The information for each patient was tabulated with demographic data and full details of epilepsy diagnosis, age at seizure onset, seizure type and frequency, and parental/caregiver interviews were included, together with antiepileptic drugs used and response to treatment. Data obtained from video-EEG recordings, brain MRI or CT scans, and neuropsychological findings were also included.

\section{Results}

We identified 22 adult patients with epilepsy and DS (11 males, 11 females) with a mean age of 46 years (range: 28-64 years). Complete evaluation of the patients was not possible, as two were lost at follow-up and two died.

The mean age at epilepsy onset was 36.8 years (range: 6-60 years). According to the proposed ILAE classification (2001), 12 patients had generalised tonic-clonic seizures, seven had focal seizures, and two had tonic seizures, in one of them the tonic seizures were triggered by sudden noise (reflex seizures). Nine patients had myoclonic seizures, predominantly involving the upper limbs, often occurring after awakening. Of those who had myoclonic seizures, one patient had eyelid myoclonia (see video sequence 1; figure 1). Of the three patients who had myoclonic status epilepticus, the status occurred at epilepsy onset in two patients. In both of these subjects myoclonic status epilepticus was easily controlled with i.v. valproate. Nine of 22 patients had focal seizures and a temporal origin was identified in some patients. Nine were classified as LOMEDS and in four patients the epilepsy was unclassified. The mean age at epilepsy onset in patients with LOMEDS was 50.2 years and in most of them cognitive decline preceded any obvious seizure onset by about $6-18$ months (5/9 subjects) or occurred at seizure onset ( $4 / 9$ subjects).

In most subjects, cognitive deterioration was too rapid for neuropsychological testing. When cognitive evaluation could be performed, the presence of memory deficits, spatial-temporal disorientation and verbal fluency impairment was detected. Parents and/or caregivers also reported loss of previously achieved daily abilities, mental slowing, behavioural disturbances such as irritability, and increased stereotyped movements.

Most of our patients were treated using monotherapy: eight with valproate, five with levetiracetam, one with phenobarbital, one with primidone and one with phenytoin. Five patients were on polytherapy because of drug resistance.

Neuroimaging (CT scan and/or MRI) was performed for 19 of 22 patients. Brain atrophy was found in seven sub- 


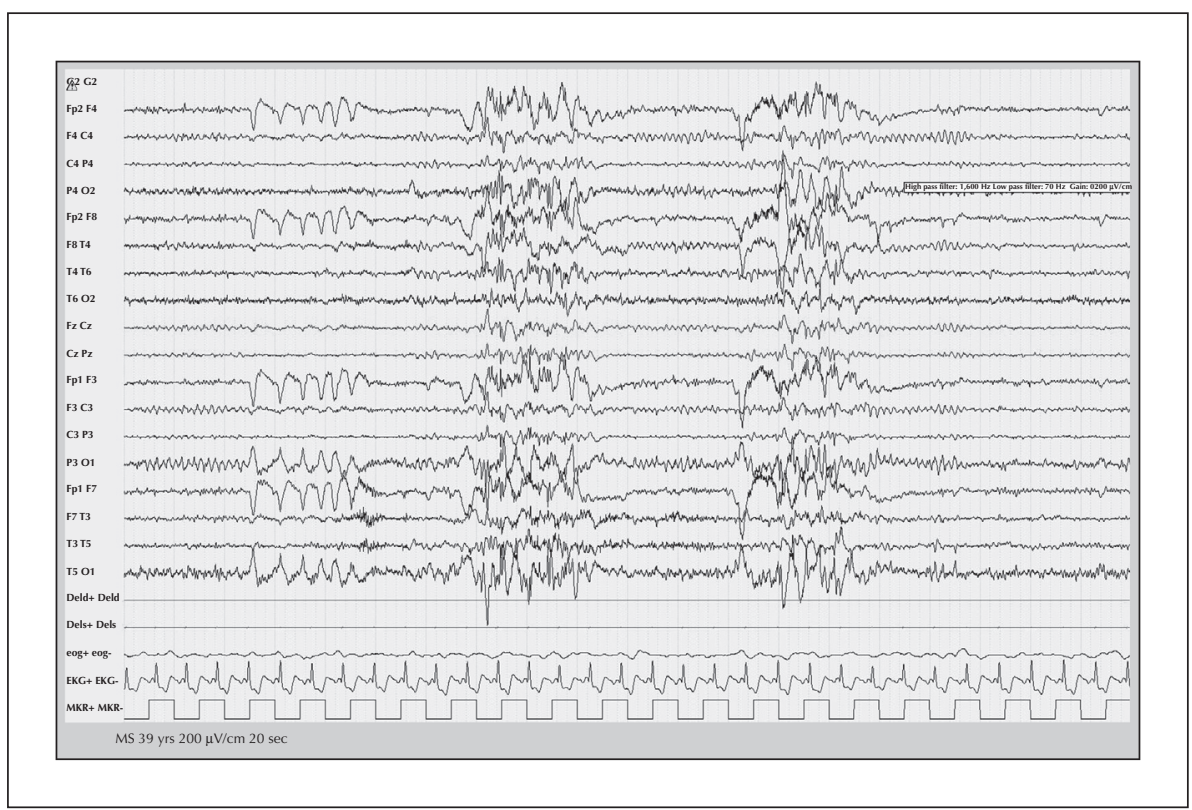

Figure 1. Diffuse polyspike waves related to eyelid myoclonia.

jects, associated with hydrocephalus in four, ischaemic lesions in one and an arachnoidal cyst in another. Basal ganglia calcifications were detected in three subjects. In eight patients the result was unremarkable.

In all patients, EEGs showed a slow and poorly organised background activity. This pattern is recognised to be typical of subjects with DS. We observed progressive slowing of the dominant background activity with increasing age. Theta activity was predominant over fronto-temporal regions in most patients (figure 2). In all except one patient with LOMEDS, bilateral polyspike-wave discharges were recorded (figure 3), mostly associated with myoclonic jerks in upper limbs (see video sequences 2 and 3; figure 4). The main clinical, EEG and neuroradiological findings of the patients are described in table 1.

\section{Discussion}

In recent decades, people with DS have lived for longer and this may be ascribed to improvements in medical care (Glasson et al., 2002). For this reason, clinical complications in adult patients with DS are becoming much more frequent.

In our sample of adult patients with DS, two main groups of epilepsy syndromes were identified: focal epilepsy, with a prevalent onset in adolescence or young adulthood, and LOMEDS, with a typical onset after the fifth decade (in five patients the onset was in the fifth decade and in four in the sixth decade). Regarding the patients with DS and focal epilepsy, the mean age of epilepsy onset was 25.7 years. In all except two, epilepsy onset occurred at a young age. In this group of patients, epilepsy was sometimes drug-resistant and continued lifelong without any clear cognitive decline. No particular neuroradiological picture was evident in this group of patients. Recently, Ferlazzo et al. (2009) described a peculiar presentation of Lennox-Gastaut syndrome in DS. In our sample, two patients had tonic seizures, but none of them were diagnosed with Lennox-Gastaut syndrome since the complete syndromic features, including multiple seizure types, characteristic EEG pattern and behavioural disturbances, were not evident.

Since the first description of LOMEDS, characterised by myoclonic epilepsy associated with dementia with onset during or after the fifth decade in DS (Genton and Paglia, 1994), few patients with this peculiar syndrome have been described in small case series (Moller et al., 2001; De Simone et al., 2006; Larner, 2007; Genton, 2007; Crespel et al., 2007). In our group of adult patients with DS and epilepsy, nine subjects were shown to fulfill the criteria for the diagnosis of LOMEDS, which represents, to our knowledge, the largest reported cohort of patients to date.

With regard to the clinical characteristics of these patients, we observed that the cognitive decline preceded any obvious seizure onset by about 6-18 months or occurred at seizure onset in most cases. As already reported (Deb et al., 2007), the early symptoms of dementia in DS patients may be difficult to recognize and the caregivers often have a crucial role in detecting the primary behavioural changes presented in the 
A. Vignoli, et al.

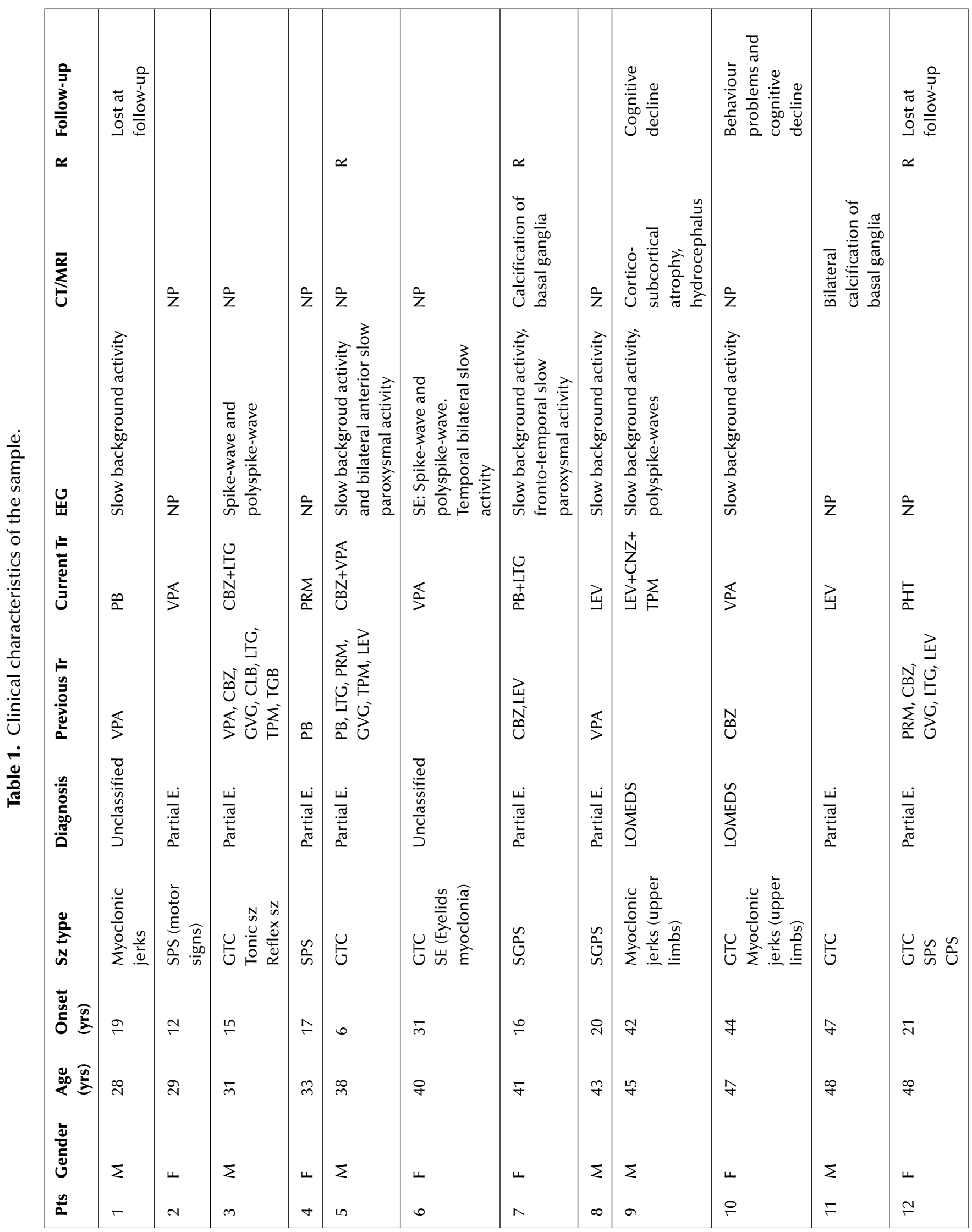




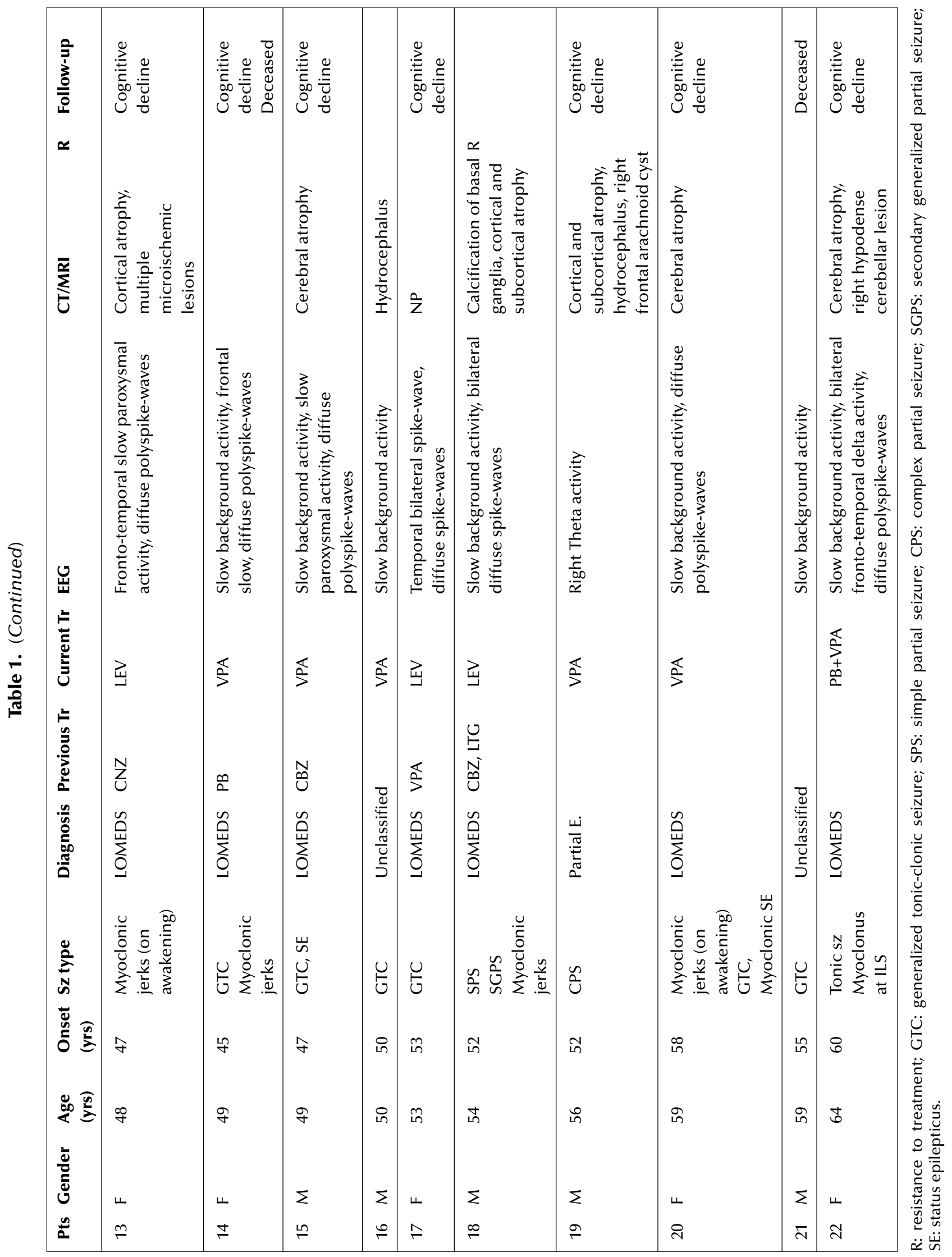




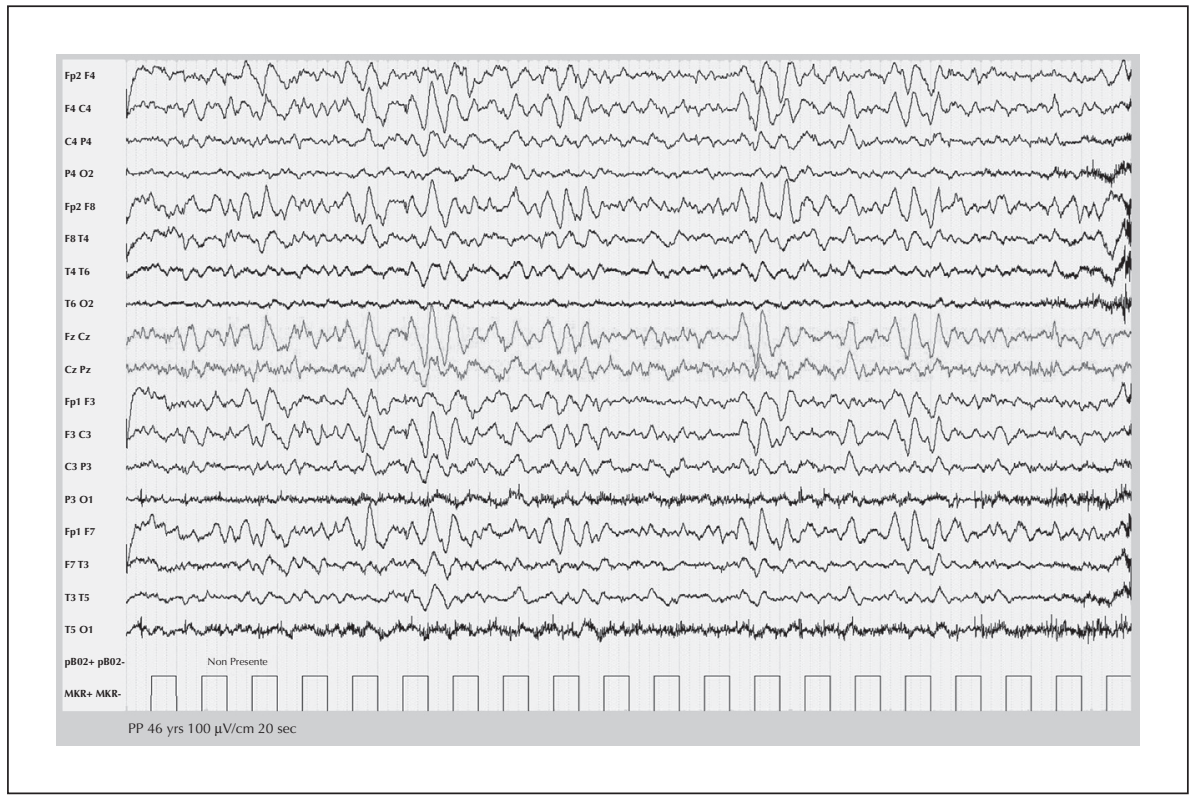

Figure 2. Slow background activity with sharp-and-slow waves with frontal predominance.

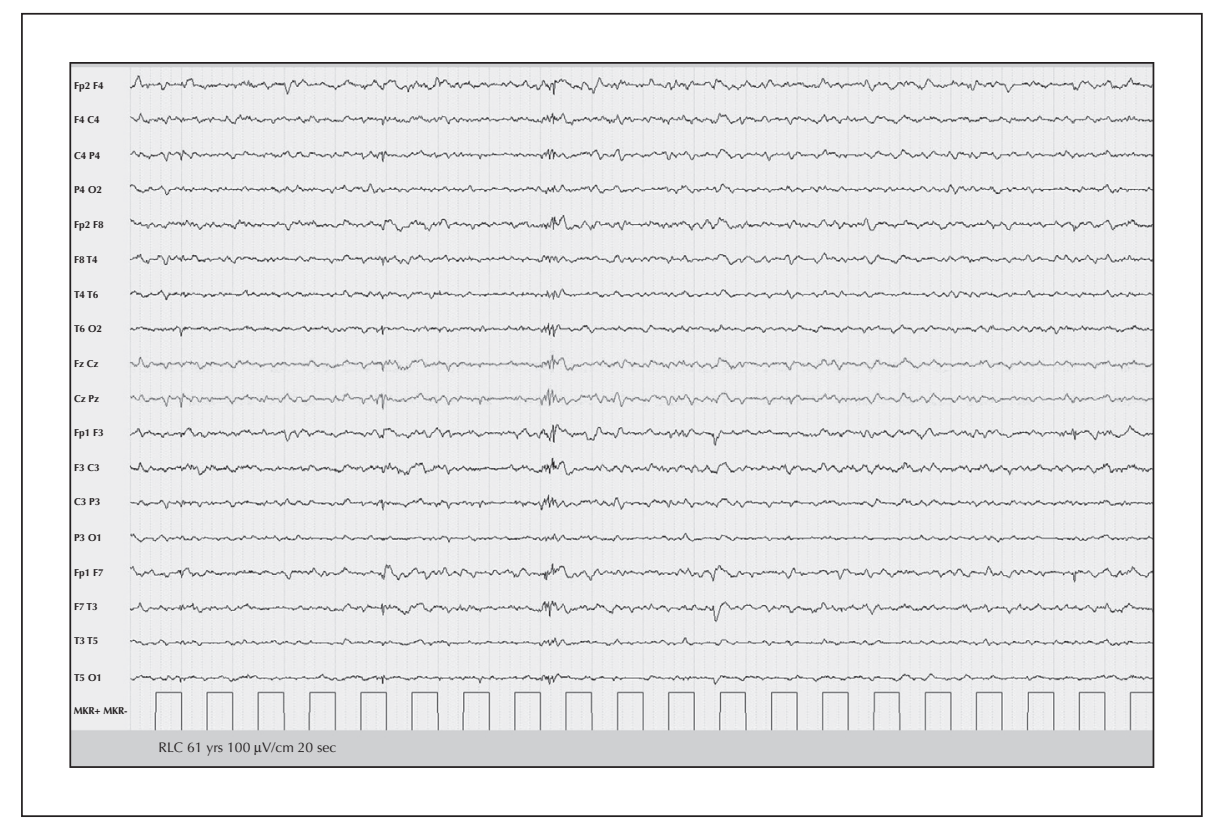

Figure 3. Diffuse slow activity with generalised polyspike waves in a patient with LOMEDS.

patients. It is noteworthy that the most frequent signs of dementia reported by the caregivers in our sample were loss of previously achieved daily abilities, mental slowing, and behavioural disturbance such as irritability and increased stereotyped movements. The clinicians should regard such early symptoms with suspicion and ask for further investigations with video polygraphic EEG recording and neuroradiological evaluation in order to diagnose LOMEDS.

The electroclinical pattern of LOMEDS was found in all patients and was characterised by slow background activity and diffuse spike waves or polyspike waves. In a few cases, it was possible to record myoclonias, mainly involving upper limbs. Neuroradiological 


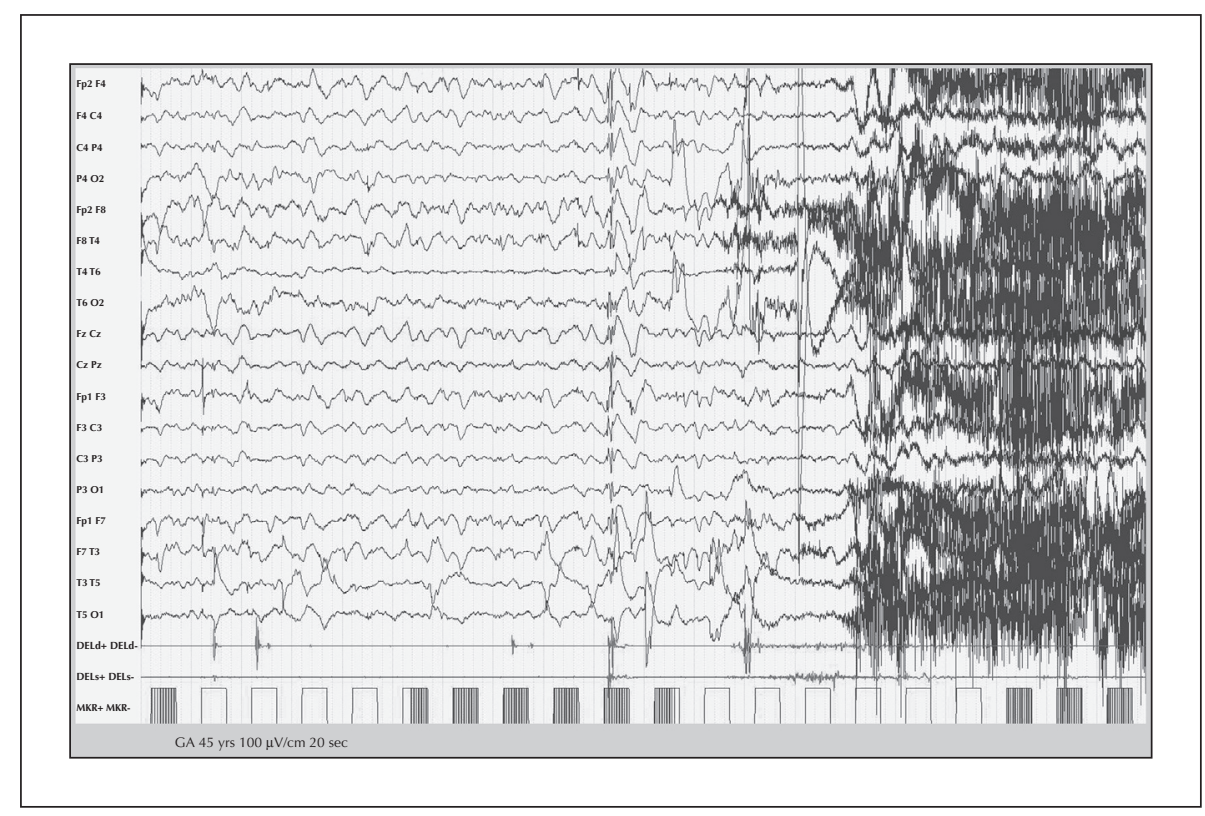

Figure 4. Myoclonic seizure induced by intermittent light stimulation in a patient with LOMEDS.

investigations in patients with LOMEDS showed only cerebral atrophy, probably related to senility. As previously reported (De Simone et al., 2006) and also in our experience, antiepileptic treatment with valproate or levetiracetam can promptly control myoclonic and generalised tonic-clonic seizures in these patients.

It is well known that the clinical picture of cognitive decline in DS is very similar to that of dementia in classic Alzheimer disease, although onset is earlier in DS. Neurophysiological analysis of myoclonus in DS showed the same characteristics present in Alzheimer disease (Wilkins et al., 1984), as did the neuropathological examination performed in a few cases. However, the incidence of epileptic seizures and myoclonus in DS patients is about eight times greater than that in Alzheimer patients (Evenhuis, 1990).

It is important to recognize the specific electroclinical pattern of LOMEDS in order to start early treatment and achieve a prompt control of the seizure disorder which may avoid further cognitive decline. Since myoclonic seizures may involve not only the upper limbs but also different parts of the body, as demonstrated in our patients with eyelids myoclonias, the full characterisation of these patients reported here should extend the description of clinical presentation and EEG pattern of epilepsy in adult patients with DS.

\section{Disclosure.}

None of the authors has any conflict of interest or financial support to disclose.

\section{Legends for video sequences}

Video sequence 1

Video-EEG recording (EEG is not presented) obtained after a generalised tonic-clonic seizure in a patient aged 39 years. Several eyelid myoclonia were recorded lasting 3-4 seconds, related to diffuse polyspike waves.

\section{Video sequence 2}

Video-EEG recording of a 45 -year-old patient presenting recurring myoclonic jerks involving both arms.

\section{Video sequence 3}

The same patient showed myoclonias induced by intermittent light stimulation.

\section{References}

Arya R, Kabra M, Gulati S. Epilepsy in children with Down syndrome. Epileptic Disord 2011; 13: 1-7.

Crespel A, Gonzalez V, Coubes P, Gelisse P. Senile myoclonic epilepsy of Genton: two cases in Down syndrome with dementia and late onset epilepsy. Epil Res 2007; 77: 165-8.

Deb S, Hare M, Prior L. Symptoms of dementia among adults with Down's syndrome: a qualitative study. J Intell Disab Res 2007; 51: 726-39.

De Simone R, Daquin G, Genton P. Senile myoclonic epilepsy in Down syndrome: a video and EEG presentation of two cases. Epileptic Disord 2006; 8: 223-37. 
Engel J. A proposed diagnostic scheme for people with epileptic seizures and with epilepsy: report of the ILAE task force on classification and terminology. Epilepsia 2001; 42: 796-803.

Evenhuis HM. The natural history of dementia in Down's syndrome. Arch Neurol 1990; 47: 263-7.

Ferlazzo E, Adjien CK, Guerrini R, et al. Lennox-Gastaut syndrome with late-onset and prominent reflex seizures in trisomy 21 patients. Epilepsia 2009; 50: 1587-95.

Genton P, Paglia G. Epilepsie myoclonique senile? Myoclonies epileptiques d'apparition tardive dans le syndrome de Down. Epilepsies 1994; 1: 5-11.

Glasson EJ, Sullivan SG, Hussain R, Petterson BA, Montgomery $\mathrm{PD}$, Bittles $\mathrm{AH}$. The changing survival profile of people with Down's syndrome: implications for genetic counselling. Clin Genet 2002; 62: 390-3.

Larner AJ. Down syndrome in the neurology clinic: Too much? Too little? Too late? Down Syndrome Research and Practice 2007; 12: 69-71.
Mc Vicker RW, Shanks OE, Mc Clelland RJ. Prevalence and associated features of epilepsy in adults with Down's syndrome. British Journal of Psychiatry 1994; 164: 528-32.

Menéndez M. Down syndrome, Alzheimer's disease and seizures. Brain and Dev 2005; 21: 246-52.

Moller JC, Hamer HM, Oertel WH, Rosenow F. Late-onset myoclonic epilepsy in Down's syndrome (LOMEDS). Seizure 2001; 10: 303-5.

Sherman SL, Allen EG, Bean LH, Freeman SB. Epidemiology of Down syndrome. Ment Retard Dev Disabil Res Rev 2007; 13: 221-7.

Smigielska-Kuzia J, Sobaniec W, Kulak W, Bockowski L. Clinical and EEG features of epilepsy in children and adolescents in Down syndrome. J Child Neurol 2009; 24: 416-20.

Wilkins DE, Hallett M, Berardelli A, Walshe T, Alvarez N. Physiologic analysis of the myoclonus of Alzheimer's disease. Neurology 1984; 34: 898-903. 